JOURNAL OF INFORMETRICS 7 (2013) 72-83

http://dx.doi.org/10.1016/j.joi.2012.08.001

\title{
Would it be possible to increase the Hirsch-index, $\pi$-index or CDS-index by increasing the number of publications or citations only by unity?
}

\author{
Péter Vinkler \\ Research Centre for Natural Sciences, Hungarian Academy of Sciences \\ H-1525 Budapest, PO BOX 17. \\ E-mail: vinkler.peter@ttk.mta.hu
}

\begin{abstract}
The aim of the study is to explore the effects of the increase in the number of publications or citations on several impact indicators by a single journal paper or citation. The possible change of the $h$-index, A-index, R-index, $\pi$-index, $\pi$-rate, Journal Paper Citedness (JPC), and Citation Distribution Score (CDS) is followed by models. Particular attention is given to the increase of the indices by a single plus citation. The results obtained by the "Successively Built-up Indicator" model show that with increasing number of citations or self-citations the indices may increase substantially.
\end{abstract}

Keywords: Hirsch-index, $\pi$-index, CDS-index, citations, self-citations, indicators

\section{Introduction}

One of the basic assumptions of scientometrics is that the citation reflects the impact of information in the publication of the cited author. If this prerequisite was regarded as invalid, no reasonable scientometric assessment could be completed.

The impact indicators of evaluative scientometrics are calculated with using the number and distribution of citations among journal papers, primarily. Recently, the most popular indices are the $h$-index (Hirsch, 2005) and h-type indices (Schreiber, 2010; Bornmann et al., 2011). The $h$-index is based on a specific selection method of the relatively (within the set) highly cited publications. Accordingly, the eminence of the scientists is characterized by the number of papers in the "elite set" (h-core papers) within their total publications. The index represents a special statistics, and it depends on the distribution of citations among the individual journal papers and on the number of the publications (see, Glänzel, 2008 and Glänzel \& Schubert, 2010). The h-type indices may represent the number of citations to the hcore papers e.g. A-index (Jin, 2006) and R-index (Jin et al., 2007) or different combinations of the rank number of papers and citations (e.g. g-index, Egghe, 2006).

In contrast to the h-type indices the $\pi$-type indices depend on the number of citations obtained to the most influential papers ("elite set", $\pi$-core) within the set analyzed. The number of $\pi$-core papers is equal to the square root of the total number of publications in the set (Vinkler, 2009, 2010a).

The CDS-index is calculated by summing up the weighted numbers of publications in different citedness categories preferring highly cited publications (Vinkler, 2011a). The CDSindex may be calculated either from the total set or only from the elite set, depending on the set studied.

The Journal Paper Citedness (JPC) index is a traditional indicator representing the aggregated citedness value (citations per paper) of the whole set analyzed. Also the Garfield (Impact) Factor represents a JPC-index. The indicator is preferably calculated by the "ratio of 
the sums" method instead of the "mean of the ratios" method (Vinkler, 2011a). The indicators investigated in the present paper are shown in Table 1.

In the present paper I try to demonstrate the effect of the gradual increase of the number of papers and citations on the indices. I will focus on the effect of a single more journal paper or citation in the set studied. In studying scientometric indicators the determination of sensitivity of the corresponding index to the changes of the measures is essential. In selecting impact indicators for evaluation purposes, the relative change of the indices to the increase in the number of citations and papers seems to be one of the most important aspects. It is especially important to study the effect of a single citation because of the possible manipulations. For decreasing the adverse effects of manipulated scientometric indicators, one has always to apply several basically different indices.

It is widely known, there is an increasing pressure on scientists to produce more and more publications and to increase their scientometric indices. $\mathrm{PhD}$ and postdoc fellows are forced to publish in journals with possible high Garfield (impact) factor, and they are keen to obtain more and more citations. Consequently, there is a growing temptation for manipulating the authorship of publications and for manipulating with citations.

Williamson (2009) summarizes the advantage of the $h$-index as follows: "it provides a single, easy to compute, quantitative measure of your cumulative impact." According to the author the simplest way to boast the Hirsch index is the application of the "Discreet System of SelfCitation (DSSC)" method. The essence of the method is as follows: those papers should be preferably referred to, which are outside the Hirsch-core, that "might be stalled or slowly approaching the "Green Line" (i.e. the citations of the $h$-level paper). According to Kotov (2010): "While the h-index does have some utility and convenience, the danger of simple numbers and unhealthy consequences of their frequent consumption... need to be remembered very well." According to the study of Bartneck \& Kokkelmans (2011) the authors can considerable inflate their $h$-index through self-citations. They propose an index ( $q$-index) to detect the possible manipulation of the $h$-index. Nevertheless, they conclude that the best strategy to attain a high $h$-index is publishing papers that are highly cited by others. However, with increasing number of publications the $h$-index may increase as well. They found an increasing difference between the "real" and manipulated $h$-index with time. More recently Ravallion \& Wagstaff (2012) conclude that the $h$-index does not correspond to some basic criteria for the scientometric impact indicators, i.e. stability (the same level for citations and influence); monotonicity (i.e. the higher the citation count the higher the influence); concavity (i.e. the influence function is concave).

I have concluded recently (Vinkler 2011b) that the relatively high $\pi$-index (Vinkler 2009, 2010a) of some researchers with only a single extreme highly cited paper could not be justified. Further, it seems to be possible i.e., to manipulate the index through consequently citing one (or several) papers in the elite set.

Through the models described in the present paper, I try to demonstrate the measure of the possible increase of the different impact indices by a single publication or citation.

\section{Possible increase of the indices by increasing the number of publications with unity while keeping the number of citations as constant}

The scientometric impact indices (Vinkler 2010b) may be sensitive or insensitive to the increase in the number of publications. A new publication has no citations at the time of publishing, it has however potential to receive citations. Nevertheless, there are impact indicators which take into account positively (e.g. CDS-index) and others which take into account negatively (e.g. C/P type indices) the publications with zero citation. The former 
method may be substantiated by the assumption that also the publications not cited may have information value.

\section{1. $\pi$-index}

The $\pi$-index characterizes the eminence of scientists (teams or journals) by the number of citations obtained to the publications in the elite set ( $\pi$-core) of the whole set of publications analyzed.

Increasing the number of publications $(P)$, it will increase the $\pi$-index only if the number of citations to the journal papers in the $\pi$-core increases through the increase of the papers. For determining the effect of a new publication on the $\pi$-index, we have to calculate the number of papers in the $\pi$-core with the increased number of the publications: $P_{\pi}=\sqrt{P+1}$. Nevertheless, we have to accept: each publication in the elite set represents an entity, which means, there are taken into account no averages, i.e. "part" papers (e.g. "2.4" papers). Because of the necessary rounding, the value of $P_{\pi}$ increases monotonously but not strictly monotonously with increasing number of total papers (P) (Table 2). It can be observed that the frequency of identical $P_{\pi}$ values, $f\left(P_{\pi}\right)$ changes according to Eq. 1:

$f\left(P_{\pi}\right)=2 P_{\pi}$

where $P_{\pi}$ is the number of papers in the $\pi$-core. Accordingly, the publication sets with $P=1$ or 2 (rounded $\sqrt{ } P=1$ ) show a $P_{\pi}$-index of unity, whereas the sets consisting of 3, 4, 5 or 6 papers will show an identical $P_{\pi}$-index $=2$ (Table 2$)$.

In calculating the $\pi$-index, the journal papers are ranked by the decreasing number of citations. Consequently, the number of citations to the $(\pi+1)$ level paper may be equal to or lower than the citations received by the $\pi$-level paper: $C_{\pi+1} \leq C_{\pi}$. If the number of papers in the $\pi$-core, $P_{\pi}$ increases by unity, the value of the modified $\pi$-index $\left(\pi_{m}\right)$ will increase by the hundredth of citations received to the $P_{\pi+1}$ paper (Eq. 2). The upper level of the increase: 0.01 $C_{\pi}$; i.e. the hundredth of citations to the $\pi$-level paper.

$\pi_{m}=\pi+0.01 C_{\pi+1}$

Accordingly, if a team has $P=110$ papers, $P_{\pi}=\sqrt{ } P=10.48 \sim 10.00$. Provided, the most highly cited 10 papers obtained together 300 citations, the $\pi$-index $=3.00$. If the team publishes one more paper $\left(P_{m}=111\right), P_{\pi m}=\sqrt{ } 111=10.54 \sim 11.00$. Provided the 11 th publication obtained 15 citations, the modified $\pi$-index will be: $\pi_{m}=3.00+0.15=3.15$.

From the data in Table 2 and 3 it follows that the $\pi$-index is highly sensitive to the increase of the number of publications at low number of papers $(P)$ and to the distribution of citations among the papers.

For three different sets of papers (A, B, C, Table 3) each consisting of the same number of publications $(P=10)$ and citations $(C=100)$ but with different distribution of citations among the papers, we may calculate different $\pi$-values $(0.92,0.70,0.31$, resp.). From the data it concludes that the $\pi$-index prefers the sets (here A) containing at least a single relatively highly cited paper over the sets with more homogeneous distribution of citations (e.g. C). With the increase of the number of papers by $3\left(\mathrm{P}_{11}, \mathrm{P}_{12}, \mathrm{P}_{13}\right.$, i.e.: $\left.\mathrm{P}_{\mathrm{m}}=13\right)$ but, keeping the number of citations at the same level $(C=100)$, the size of the elite set, $P_{\pi}$ increases from 3 to $4(\sqrt{ } 13=3.6 \sim 4)$. Accordingly, the increase of the $\pi$-index will be the higher, the higher the number of citations to the paper ranked as $(\pi+1)$. The increase of the $\pi$-index is highest for 
set C $(29.03 \%)$ where the citedness of the $(\pi+1)$-level paper (10) is highest. At higher $P$ values (e.g. between 21-42 papers) the size of the elite set does not change while increasing the size of the set by $35.48 \%$. Consequently, neither the $\pi$-index changes provided of course that the citedness of the papers in the elite set remains at the earlier level. Naturally, increasing the number of publications from 42 to 43 also the size of the elite set increases (from 6 to 7). Accordingly, the $\pi$-index will increase with the hundredth of citations obtained to the 7 th paper.

\section{2. $C D S$-index}

The calculation of the CDS-index for individuals takes into account also the journal papers without citation. Consequently, each new journal paper published increases the CDSindex by unity, according to the weighting method applied here (Table $1 ; \mathrm{w}_{1}=1, \mathrm{w}_{2}=2, \mathrm{w}_{3}=$ $3 \ldots)$. At the time of publishing, the article has generally no citations. Later, after having obtained two citations at least, the paper will get into the 2 nd citation category with two scores (see Table 4). The relative increase of the CDS-index may be high at low CDS values (or low number of publications). By publishing a new paper, the relative change of the index is similar to that given for the change in citations (see later).

\subsection{Journal Paper Citedness (JPC)}

The JPC $=C / P$ index represents an aggregate indicator (i.e., $C$ and $P$ refer to the whole set studied) consequently, increasing the number of papers by publishing a new paper, the value of the index decreases. The measure of the decrease depends on the size of the set analyzed. The sets consisting of low number of papers are naturally more sensitive to increasing the denominator (e.g. $P=1 ; C=10 ;(C / P)=10$, and $P=2 ; C=10 ;(C / P)=5.0$, whereas $P=10 ; C=10 ;(C / P)=1.0$ and $P=11 ; C=10,(C / P)=0.9)$. Naturally, it is true that a positive linear relationship exists between the total numbers of citations obtained and total number of papers published, in general.

\subsection{Hirsch index (h-index) and Hirsch-type indices}

The Hirsch index may be regarded as the number of publications in the elite set of the whole set studied. Accordingly, the eminence of scientists (teams or journals) is characterized by the number of publications in a selected subset.

Increasing the number of papers in the whole set analyzed, whereas keeping the number of citations constant, the value of the $h$-index will not change.

The publication of the first paper $\left(P_{l}\right)$ of an author results in arriving at the level of $h=1$ only after $P_{l}$ having received its first citation (Table 5, A). To increase the $h$-index $=1$ by unity $(h=2)$, one need to publish a second paper $\left(P_{2}\right)$, to obtain one more citation to $P_{1}$ at least, and to receive two citations (at least) also to $P_{2}\left(\Sigma C_{\min }=4\right)$. In this case "there are two papers each having obtained two citations, at least" (Table $5, \mathrm{~B}, \mathrm{~b}$ ). It is obvious that the minimum number of total citations $\left(\Sigma C_{\text {min }}\right)$ obtained by a set with $h$-index $=\mathrm{n}$, is $\sum C_{\text {min }}=\mathrm{n}^{2}$. It is also obvious that neither the A-index nor R-index will change by increasing the number of publications and keeping the number of citations as constant.

\section{Possible increase of the impact indices by obtaining more citations while keeping the number of papers as constant}

The value of the impact indicators studied may increase by increasing the number of citations obtained, in general. The possible relative increase of the individual indices is 
different and it strongly depends on the size of the publication set analyzed. The increase of the indices studied by increasing the number of citations with unity is demonstrated in Table 6.

\section{1. $\pi$-index}

The $\pi$-index is sensitive to the increase in the number of citations only by obtaining plus citation(s) to the publication(s) in the $\pi$-core (Table 6). Citations to papers outside the $\pi$-core, do not influence the $\pi$-index. If a paper in the $\pi$-core obtains one more citation, the $\pi$-index will increase by 0.01 (e.g. $\pi=1.20 ; \pi_{m}=1.21$ ). The goal of applying the index is to measure the growth impact of the most influential publications. Therefore, it is readily acceptable that only the citations to the relatively highly cited papers increase the index. The relative percentage increase takes $10 \%$ if the $\pi$-core contains papers with a total of 10 citations ( $\left.\pi=0.10, \pi_{m}=0.11\right)$. At higher number of citations to the $\pi$-core papers (e.g. $100, \pi=1.00$ ), the increase by a single plus citation is significantly less $\left(1 \% ; \pi_{m}=1.01\right)$. At 300 citations $(\pi$ $=3.00)$ the increase of citations by unity $\left(C_{m}=301\right)$ modifies the index of $0.33 \%$ only $\left(\pi_{m}=\right.$ 3.01). The linear increase of the $\pi$-index by the increase of citations to the papers in the $\pi$-core can be given by Eq. 3, where $C_{m}$ is the number of the new citations received by the $\pi$-core papers.

$\pi_{m}=\pi+0.01 C_{m}$

\subsection{CDS-index}

The CDS-index is sensitive to the change in the citedness of any papers in the set analyzed, provided: the paper obtains at least one more citation than the upper limit of any category, as this way it will get into a higher citedness category (Table 4). If a paper, e.g. with one citation in the first category (citations: $0-1$ ) obtains one more citation, it will get into the 2nd class (citations: 2-4) and the CDScore will increase from 1 to 2. Changing in citedness within a given citedness category, it does not change the CDS-index (e.g. a paper with increasing the number of citations from 9 up to 16, will get the same score, 4 ). If however, the number of citations arrives at the level of 17, the CDScore of the paper will increase by unity (from 4 to 5). The relative percentage increase of the CDS-index may be extremely high by increasing the number of citations by unity if the number and/or citedness of the papers is low (Table 4), and it will be significantly lower with higher $P$ and $C$ values (e.g. CDS $=50.00$ and $\mathrm{CDS}_{\mathrm{m}}=51.00$, which corresponds to an increase of $2 \%$ ) (Table 6).

If we keep the number of papers as constant whereas the number of citations to a paper increases by unity and it will get this way to a higher category, the CDS-index would increase also by unity:

$\Delta \mathrm{CDS}=\left(\mathrm{r}_{\mathrm{i}}+1\right)-\mathrm{r}_{\mathrm{i}}=1$

where $r_{i}$ is the rank number of the category. Eq 4 is valid only if the weighting factor of the papers in the categories is equal to the rank number. Naturally, with applying weighting factors $\mathrm{w}_{\mathrm{i}} \neq 0$ for the rank categories, we have to calculate with ( $\left.\mathrm{w}_{\mathrm{i}} \mathrm{r}_{\mathrm{i}}\right)$ (Vinkler, 2012).

\subsection{Hirsch index (h-index)}

It has been proved earlier (Vinkler, 2007, 2010a) that the h-index can be enhanced by unity through receiving only a single citation. The publication sets of which $h$-index can be 
increased by unity through increasing the number of citations by unity, have been termed as anomalous Hirsch sets. The sets with the possibility of increasing the $h$-index by unity through two further citations have been named as specific Hirsch sets. Table 5 shows some simple $h$-sets as models. The journal papers are ranked by the decreasing number of citations. If the first paper in the set obtains one or any number of citations higher than unity $\left(c_{1} \geq 1\right)$ and the second paper obtains zero or one citation $\left(c_{2}=0\right.$ or 1$)$, the $h$-index will be equal to unity (Table 5, A, a). Naturally, the third publication (if any) in the set may figure with zero or also with one citation. If however the first paper figures with 2 citations and the second paper in rank with one citation (Table 5, B, a), and the second paper will receive a single more citation $\left(c_{2}=2\right)$ (Table 5, B, b), there will be two papers in the set with two citations each, consequently the $h$-index will increase to 2 . The situation is similar for sets with $h=2,3$, etc. (Table 5, C, D).

Table 7 gives some model examples for the possible increase of the $h$-index in anomalous (A, B) and specific (C, D) Hirsch-sets. The criteria for the increase of the $h$-index by unity in an anomalous $h$-set may be given as follows:

$\mathrm{c}_{\mathrm{h}}=\mathrm{h}+\mathrm{n} \leq \mathrm{c}_{(\mathrm{h}-1)} \quad$ and $\quad \mathrm{c}_{(\mathrm{h}+1)}=\mathrm{h}$

where $c_{h}$, is the number of citations obtained by the paper of which rank number is equal to the $h$-index, $n$ is a positive integer, and $h$ is the $h$-index of the set. Accordingly, the number of citations obtained by the $h$-paper $\left(c_{h}\right)$ should be higher by unity than the $h$-index at least and, the number of citations to the paper next in rank $(h+1)$ should be equal to the $h$-index (A and B, Table 7).

For specific Hirsch sets the increase of the $h$-index may be attained by obtaining two further citations (C and D, Table 7). Accordingly, the criteria of the citedness for increasing the $h$-index by unity for a specific Hirsch-set:

Either:

$\mathrm{c}_{\mathrm{h}}=\mathrm{h}=\mathrm{c}_{(\mathrm{h}+1)}<\mathrm{c}_{(\mathrm{h}-1)}$

Or:

$\mathrm{c}_{\mathrm{h}}=\mathrm{h}+\mathrm{n} \leq \mathrm{c}_{(\mathrm{h}-1)} \quad$ and $\quad \mathrm{c}_{(\mathrm{h}+1)}=\mathrm{h}-\mathrm{n}$

Naturally, it is valid that all papers with ranking lower than $h$ should have citations equal to $h$, at least. Increasing the citations to the $(h+1)$ paper (anomalous Hirsch-set: A, B) by unity, we obtain $h=6$ for both sets (A' and B'). With the increase of citations to the paper ranked as the $h$-number and the paper ranked as $(h+1)$ each by unity (specific Hirsch set, C), the $h$-index increases from 5 to 6 (C'). Similarly, with increasing the number of citations to the $(h+1)$ paper (D) by two, the $h$-index will increase to 6 .

It should be mentioned that the $h$-index is not-sensitive to increasing the number of citations to papers in the Hirsch core, although these publications may be regarded as the most influential publications in the corresponding set.

\subsection{A-index}

The A-index (Table 1) may be regarded as an improved $h$-index because it takes into account the increasing influence of the $h$-core papers manifested by the increased number of 
citations (Jin, 2006, Jin et al., 2007). It increases namely with increasing citations to the $h$ core publications.

The effect of a single citation, on the A-index, is shown by a simple model. It is supposed (Table 8) that the plus citation will be received by the most cited paper in the $h$-core. The data in Table 8 reveal, the A-index may increase from 1.0 to $2.0\left(\mathrm{~A} / 1 \mathrm{vs} \mathrm{A}^{\prime} / 1\right)$ and from 2.0 to 2.5 $\left(\mathrm{B} / 1 \mathrm{vs} \mathrm{B}^{\prime} / 1\right)$. It is obvious that the value of the index increases relatively rapidly with obtaining a single more citation for sets of low A value. The percentage increase seems to be, however negligible from $\mathrm{A}=10$ (Table 6).

\section{5. $R$-index}

Also the $R$-index may be regarded as an improvement of the $h$-index (Jin, 2006; Jin et al., 2007). The assumption behind the index is that it would represent the scientific eminence by the square root of the total impact (i.e. number of citations) of the publications in the elite set selected as the $h$-core. Accordingly, each further citation to the $h$-core publications will enhance the index.

The models in Tables 6 and 8 may demonstrate the effect of a single plus citation to the $h$ core publications on the $R$-index. It is supposed that the plus citation will be obtained by the first ranked paper.

According to the data in Tables 6 and 8 the percentage change of increase of the $R$-index is great at low $R$ values but from about $R=5$, the increase will be negligible.

\subsection{Journal Paper Citedness (JPC)}

Each citation to any paper in the set analyzed increases the value of the index, which is regarded as a global or aggregate indicator. It is obvious that the increase in citations even by unity, whereas the number of papers is kept constant, will increase the index significantly (e.g. by $100 \%$ ) at low citedness values (e.g. at 0.1 citations/paper). The change is many lower (e.g. $0.2 \%$ ) at high citation rate (50 citations/paper) (Table 6).

Table 6 summerizes the influence of a single plus citation on the value of the indices studied. The original value of the indicators is given within a very long range, i.e. from 0.10 up to 200.00 for $\pi$ and JPC. The effect on CDS, $h, \mathrm{~A}$ and $\mathrm{R}$ is given between the values of 1.00 and 200.00 .

The general trend is obvious: the increase of the indices as a consequence of a single new citation is the greater the lower their original value, at least in the framework of the model examples. This feature draws our attention to the scientists at an early stage of their carrier who may increase their records with only a single journal paper or several citations, substantially.

Note, that within the conditions used for the calculation, the CDS and $h$-index may show the greatest changes at low values of the indices. It is obvious that the JPC indicator is also very sensitive to the changes in the number of citations or papers at low number of publications or citations.

\section{The effect of successive citations on the indicators studied}

To demonstrate the possible effect of successively citing publications on some scientometric impact indicators, a model experiment was performed. The preconditions to the model applied are the followings: 
- each proceeding paper of a selected set of papers refers to each preceding paper in the same set, and

- no citations from other sources are taken into account.

The fulfillment of the former requirement may be termed as the principle of the "successively built-up indicator". The conditions mentioned may be fulfilled by self-citations most easily.

The mean number of references in journal papers was found 36.76 in biology and biochemistry, and 28.64, 24.11, and 18.25 in chemistry, physics and mathematics, respectively (Vieira \& Gomes 2010). Accordingly, it would not be surprising to find 10-15 references in an article to the earlier papers of the same author(s). Let us take a set of publications consisting of $P=10$ papers which are referenced according to the principle of the Successively Built-up Indicator model (SBI-model) (Table 9). We may suppose that the journal papers in the set of an author or group of authors studied obtain no citations from other authors and, each year only a single paper is published. Accordingly the set selected obtains a total of 45 citations $(\Sigma \mathrm{C}=[\mathrm{P}(\mathrm{P}-1)] / 2)$ in 10 years (where $P$ is the total number of papers).

In Table 9 each " 1 " represents a reference given by the referencing publication (e.g. $\left.P_{4}\right)$ to one of the preceding publications (i.e. $\left.P_{l}\right)$. Accordingly, in the 4th year of the period $(P=4)$ studied $P_{1}$ obtained altogether $C C\left(P_{1}\right)=3$ citations, whereas $P_{2}$ obtained $C C\left(P_{2}\right)=2$ citations, $P_{3}$ obtained $C C\left(P_{3}\right)=1$ citation and $P_{4}$ zero citation. Consequently, the $h$-index of the set of papers $\left(P_{1}, P_{2}, P_{3}, P_{4}\right)$ is 2 this year, because there are two papers each with 2 citations, at least (i.e. one with 2 and one with 3 citations) but, there are no three papers with 3 or more citations each. The number of papers in the $\pi$-core: $\sqrt{ } 4=2$, accordingly, the $\pi$-index $=0.01$ ( 3 $+2)=0.05$. The CDS-index $=6$, because there are 2 papers in the 1 st $(C=0-1)$ and 2 papers in the 2 nd citedness category $(C=2-4)$. Accordingly, CDS $=2+(2 \cdot 2)=6$. The $\pi$-rate index: $C\left(P_{\pi}\right) / P_{\pi}=5 / 2=2.50$. The A-index which refers to the average impact of the $h$-core publications shows a similar value (2.50). However, at higher number of publications, there is a significant difference between the value of the indices (e.g. $P=10 ; \mathrm{A}=7.00$ and $\pi$-rate $=$ 8.00). The $R^{2}=C\left(P_{h}\right)$-index represents a similar value (5.02) as 100 times $\pi$-index $=5.0$ for the set consisting of 4 papers. For the set with 10 papers the difference between the indices is significant $\left(100 \pi=24<\mathrm{R}^{2}=35.05\right)$. The discrepancy can be attributed to the different growth of the $h$-core and $\pi$-core.

From the model in Table 9 it concludes that the $h$-index for even and uneven number of papers ( $P_{e}$ and $P_{u}$, respectively) may be calculated according to Eq. 8 and 9 , respectively.

$h=\frac{P_{e}}{2}$

$h=\frac{P_{u}}{2}-0.5$

From Eq. 8 and 9 it concludes that a set consisting of e.g. 7 papers, which corresponds to the successively built-up indicator (SBI) principle, may show an $h$-index of 3 , and for sets with 10 or 20 papers the $h$-index is equal to 5 or 10, respectively. These values are rather high considering that a senior research staff member may show an $h$-index of about $15-25$ in chemistry (Vinkler 2010a, 2011b). 
The indicator/year relations can be approximated by linear functions. The increase of the impact indices in time by the SBI-model (Table 9) seems to be highest for the CDS-index. (The slope of the CDS/year function is 2.58 , whereas that for A, R, JPC, $h$ and $\pi: 0.77,0.64$, $0.50,0.52,0.02$, resp.)

Several scientometric impact indicators were calculated for 15 junior and 15 senior scientists for comparison. The mean publication life time (PLT) of senior and junior scientists was found as 8.60 and 32.30, in 1975-2010, respectively (PLT $=2011$ - year of the first publication in WoS since 1975). Highly significant differences were found between the means of the indices of the two groups of scientists, except for the $(C / P)$ index (Table 10).

To obtain information on the measure of the successively built-up indicators during a period of 10 and 20 years (or more precisely: after having published 10 or 20 papers), the indicators calculated by the model (Table 9) were related to the indicators of the junior (before $\mathrm{PhD}$ ) and senior (having obtained $\mathrm{PhD}$ ) authors active in the research institutes of the Research Centre for Natural Sciences of the Hungarian Academy of Sciences. The data in Table 10 reveal that a researcher having published 10 or 20 papers which are cited only by his or her own publications may attain $15.14 \%$ or $63.91 \%$, of total citations of junior scientists, respectively. By the end of a period with 20 papers the $h$-index and CDS-index attained by the SBI-technique may even surpass that of junior scientists (116.28\% vs. $122.26 \%$, resp.). Naturally, the percentage rates are significantly lower using the group of senior researchers as standard.

From the data in Table 10 it may be concluded that the CDS-index, $h$-index and R-index could be influenced with high efficiency. Only with 10 papers and self-citations $58.14 \%$ of the $h$-index and $43.80 \%$ of the CDS-index of the junior researchers $(h=8.60 ; \mathrm{CDS}=54.80)$ could be attained. The measure of increase of the indices is different by time for the different impact indicators.

The Pearson correlation coefficients between the indicators studied (Table 11) show that the $P, C, h, \mathrm{~A}, \mathrm{R}$ and $\pi$-index correlate closely with each other. (Since the indicators are not normally distributed, the Spearman correlation coefficients would seem to be more appropriate. However, the Spearman coefficients yield no plus information, here.) The mentioned indices characterize different aspects of the publication sets. The interrelatedness of the mentioned indices may validate each other. The observation corresponds to the principle of the "converging validation of scientometric indicators" (Vinkler, 2011b). According to this principle the already accepted and widely used indices (e.g. $P, C$ and $h$ ) may verify the application of the newly introduced indices $(A, R, \pi)$. Note, the aggregate citedness index $(C / P)_{t}$ shows significant correlation neither with $P$ nor with CDS. Nevertheless, it correlates with the A-index and $\pi$-rate significantly $(\mathrm{r}=0.75$ and $\mathrm{r}=0.89$, respectively). The citation rate values of the relatively highly cited papers in the elite sets (papers in the $h$-core and $\pi$-core), i.e. the A-index and $\pi$-rate, respectively, show an excellent correlation with each other $(\mathrm{r}=0.95)$. Similarly, the indices $(\mathrm{R}$ and $\pi)$, which represent the growth impact of the elite sets (i.e. $h$-core and $\pi$-core, resp.) show a very close correlation $(\mathrm{r}=0.97)$. The correlation between the number of papers $(P)$ and A-index is low but significant $(\mathrm{r}=0.58)$, the $\pi$-rate and $P$ shows no significant correlation $(\mathrm{r}=0.37)$.

\section{Conclusions}

The results of the study show that the scientometric impact indicators may be influenced even by a single new publication or citation significantly.

In the present paper only some indicators are studied. Another important impact indices and methods are planned to investigate in the near future, e.g. $g$-index (Egghe, 2006), highly 
cited papers (Aksnes, 2003; Aksnes \& Taxt, 2004), percentile distribution of publications by citation (Bornmann \& Mutz, 2011; Leydesdorff \& Opthof, 2011).

The results attained with the "Successively Built-up Indicator" model presented here would indicate the need of the introduction of comprehensive publication assessment methods with peer reviews instead of the mechanical application of some computerized scientometric indices. The possible adverse effects of self-citations on the indices ought to be avoided (see Costas et al., 2010; Schreiber, 2008, 2009). The manipulation with the number of publications and citations may endanger the fair assessment of publications of scientists or teams. The impact of the possible manipulation seems to be significantly greater for sets consisting of low number of publications and citations.

The incorrect use of the evaluation indices, incompleteness of data banks, and automatic application of the evaluation methods whereas neglecting e.g. the difference in the bibliometric characteristics of subject fields may cause severe errors in the publication assessment. Scientometricians, included reviewers of the scientometric journals may do a lot for improving the situation with determining (or with recommending to determine) validity, reliability and applicability of the assessment indicators suggested or used. In constructing relevant indicators, always the aim and function of their application should orientate the selection of the relevant scientometric elements, units, time-periods analyzed, and calculation methods used (Vinkler, 2001, 2010b). The possible errors in the indices make it necessary to apply several indices instead of relying on a single one. 


\section{References}

Aksnes, D. W. (2003). Characteristics of highly cited papers. Research Evaluation, 12, 159170.

Aksnes, D. W. \& Taxt, R. E. (2004). Peer reviews and bibliometric indicators: a comparative study at a Norwegian university. Research Evaluation, 13, 33-41.

Bartneck, C., \& Kokkelmans, S. (2011). Detecting $h$-index manipulation through self-citation analysis. Scientometrics, 87, 85-98.

Bornmann, L., \& Mutz, R. (2011). Letter to the Editor. Journal of Informetrics, 5, 228-230.

Bornmann, L., Mutz, R., Hug, S. E., \& Daniel, H-D. (2011). A multilevel meta-analysis of studies reporting correlations between the $h$ index and 37 different $h$ index variants. Journal of Informetrics, 5, 346-359.

Costas, R., van Leeuwen, T. N., \& Bordons, M. (2010). Self-citations at the meso and individual levels: Effects of different calculation methods. Scientometrics, 82, 517-537.

Egghe, L. (2006). Theory and practice of the g-index. Scientometrics, 69, 131-152.

Glänzel, W. (2008). On some new applications of statistics related to the h-index. Scientometrics, 77, 187-196.

Glänzel, W., \& Schubert, A. (2010). Hirsch-type characteristics of the tail of distributions. The generalised h-index. Journal of Informetrics, 4, 118-123.

Hirsch, J. E. (2005). An index to quantify an individual's scientific research output. Proceedings of the National Academy of Sciences of the United States of America, 102, 16569-16572.

Jin, B. (2006). h-index: An evaluation indicator proposed by scientist. Science Focus, 1, 8-9.

Jin, B., Liang, L., Rousseau, R. \& Egghe, L. (2007). The R- and AR-indices: Complementing the h-index. Chinese Science Bulletin, 52, 855-863.

Kotov, N. A. (2010). Fraud, the $h$-index, and Pasternak. ACS Nano, 4, 585-586.

Leydesdorff, L., \& Opthof, T. (2011). Remaining problems with the „new crown indicator” (MNCS) of the CWTS. Journal of Informetrics, 5, 224-225.

Ravallion, M., \& Wagstaff A. (2012). On measuring scholarly influence by citations. Scientometrics, 88, 321-337.

Schreiber, M. (2008). The influence of self-citation corrections on Egghe's g-index. Scientometrics, 76, 187-200.

Schreiber, M. (2009). The influence of self-citation corrections and the fractionalized counting of multi-authored manuscripts on the Hirsch index. Annalen der Physik (Berlin), $18,607-621$. 
Schreiber, M. (2010). Twenty Hirsch index variants and other indicators giving more or less preference to highly cited papers. Annalen der Physik (Berlin), 522, 536-554.

Vieira, E. S., \& Gomes, J. A. N. F. (2010). Citations to scientific articles: Its distribution and dependence of the article features. Journal of Informetrics, 4, 1-13.

Vinkler, P. (2001). An attempt for defining some basic categories of scientometrics and classifying the indicators of evaluative scientometrics. Scientometrics, 50, 539-544.

Vinkler, P. (2007). Eminence of scientists in the light of the h-index and other scientometric indicators. Journal of Information Science, 33, 481-91.

Vinkler, P. (2009). The $\pi$-index: a new indicator for assessing scientific impact. Journal of Information Science, 35, 602-612.

Vinkler, P. (2010a). The $\pi_{\mathrm{v}}$-index: a new indicator to characterize the impact of journals. Scientometrics, 82, 461-475.

Vinkler, P. (2010b). The Evaluation of Research by Scientometric Indicators (pp. 1-313). Oxford: Chandos Publishing.

Vinkler P. (2011a). The case of scientometricians with the ,absolute relative” impact indicator. Journal of Informetrics, 6(2): 254-264.

Vinkler, P. (2011b). Application of the distribution of citations among publications in scientometric evaluations. Journal of the American Society for Information Science and Technology, 62, 1963-1978.

Vinkler, P. (2012). The Garfield impact factor, one of the fundamental indicators in scientometrics. Scientometrics, DOI: 101007/s11192-012-0688-7

Williamson, J. R. (2009). My $h$-index turns 40: my midlife crisis of impact. ACS Chemical Biology, 4, 311-313. 


\section{Table 1}

The scientometric impact indicators studied.

\begin{tabular}{|c|c|c|}
\hline Name and acronym & The index characterizes & Calculation method \\
\hline $\begin{array}{l}\text { Hirsch index, } \\
h \text {-index } \\
\text { (Hirsch, 2005) }\end{array}$ & $\begin{array}{l}\text { Eminence of a set of } \\
\text { publications both } \\
\text { qualitatively and } \\
\text { quantitatively. }\end{array}$ & $\begin{array}{l}\text { Hirsch index: the highest rank }\left(r_{h}\right) \text { of } \\
\text { the paper to which the number of } \\
\text { citations obtained is equal to or higher } \\
\text { than its rank number (and, there is no } \\
\left(r_{h+1}\right) \text { paper with }\left(r_{h+1}\right) \text { citations). The } \\
\text { papers are ranked by the decreasing } \\
\text { number of citations. }\end{array}$ \\
\hline $\begin{array}{l}\text { Number of } h \text {-core } \\
\text { papers, } P_{h}(h \text {-level })\end{array}$ & $\begin{array}{l}\text { Number of the most } \\
\text { significant papers within } \\
\text { the corresponding set. }\end{array}$ & $\begin{array}{l}\text { Number of the papers in the } h \text {-core (i.e. } \\
\text { the papers from } r_{l} \text { to } r_{h} \text { ) }\end{array}$ \\
\hline $\begin{array}{l}\text { A-index } \\
\text { (Jin, 2006) }\end{array}$ & $\begin{array}{l}\text { Mean specific impact of } \\
\text { the publications in the } \\
\text { Hirsch-core }\left(P_{h}\right) \text {. }\end{array}$ & $\begin{array}{l}A=\frac{C\left(P_{h}\right)}{h} \\
\text { where } C\left(P_{h}\right) \text { is the number of citations } \\
\text { obtained by } P_{h} \text { papers. }\end{array}$ \\
\hline
\end{tabular}

\begin{tabular}{|c|c|c|}
\hline $\begin{array}{l}\text { R-index } \\
\text { (Jin et al., 2007) }\end{array}$ & $\begin{array}{l}\text { Gross impact of the } h- \\
\text { core papers. }\end{array}$ & $R=\sqrt{C\left(P_{h}\right)}$ \\
\hline $\begin{array}{l}\pi \text {-index } \\
\text { (Vinkler, 2009) }\end{array}$ & $\begin{array}{l}\text { Eminence of a set of } \\
\text { publications both } \\
\text { qualitatively and } \\
\text { quantitatively. }\end{array}$ & $\pi$-index $=0.01 C\left(P_{\pi}\right)$ \\
\hline$\pi$-core citations, $C\left(P_{\pi}\right)$ & $\begin{array}{l}\text { Total impact of } \\
\text { publications in the elite } \\
\text { set, } P_{\pi}\end{array}$ & Number of citations to $P_{\pi}$ papers. \\
\hline $\begin{array}{l}\text { xumber of the } \\
\text { publications in the } \pi \text { - } \\
\text { core, } P_{\pi}\end{array}$ & $\begin{array}{l}\text { Number of the most } \\
\text { influential papers within } \\
\text { the corresponding set. }\end{array}$ & $\begin{array}{l}P_{\pi}=\sqrt{ } P \\
P: \text { total number of papers. } \\
\text { The papers are ranked by decreasing } \\
\text { number of citations. }\end{array}$ \\
\hline$\pi$-rate & $\begin{array}{l}\text { Specific impact of the } \pi \text { - } \\
\text { core papers. }\end{array}$ & $\begin{array}{l}\pi \text {-rate }=\frac{C\left(P_{\pi}\right)}{P_{\pi}} \\
\text { Citations to papers in the elite set } \\
\text { ( } \pi \text {-core) divided by the number of } P_{\pi} \text {. } \\
\text { papers. }\end{array}$ \\
\hline $\begin{array}{l}\text { Citation Distribution } \\
\text { Score, } \\
\text { CDS-index } \\
\text { (Vinkler, 2011b) }\end{array}$ & $\begin{array}{l}\text { Eminence of a set }{ }^{*} \text { of } \\
\text { publications both } \\
\text { qualitatively and } \\
\text { quantitatively. }\end{array}$ & $\begin{array}{l}\text { CDS }=w_{1} P_{1}+w_{2} P_{2}+\ldots+w_{14} P_{14} \\
\text { where, } w_{1}, w_{2} \ldots w_{14} \text { are weights and } P_{1}, \\
P_{2} \ldots \text { are the number of papers in the } \\
\text { corresponding citedness category (see } \\
\text { Table } 2 \text { ). }\end{array}$ \\
\hline $\begin{array}{l}\text { Journal Paper Citedness, } \\
(C / P)_{t}\end{array}$ & $\begin{array}{l}\text { Specific impact of a set } \\
\text { of journal papers. }\end{array}$ & $\begin{array}{l}\text { The number of citations }(C) \text { obtained to } \\
(P) \text { papers in the set divided by the } \\
\text { number of papers }(P) \text {. }\end{array}$ \\
\hline
\end{tabular}


$\mathrm{x}$ : for sets consisting of high number of publications (e.g. journals), the $\pi_{\mathrm{v}}$-index can be preferably applied: $\pi_{\mathrm{v}}=(10 \log P-10)$.

": as "set" the total number of papers $(P)$ analyzed or the number of papers in the $\pi$-core $\left(P_{\pi}\right)$ may be understood. 


\section{Table 2}

Calculating the number of publications in the $\pi$-core $\left(P_{\pi}\right)$.

\begin{tabular}{|c|c|c|c|c|c|c|c|c|c|c|c|c|c|c|}
\hline$P$ & 1 & 2 & 3 & 4 & 5 & 6 & 7 & 8 & 9 & 10 & 11 & 12 & 13 & $14 \ldots$ \\
\hline$\sqrt{ } P$ & 1 & 1.41 & 1.73 & 2.00 & 2.24 & 2.45 & 2.65 & 2.83 & 3.00 & 3.16 & 3.32 & 3.46 & 3.61 & 3.74 \\
\hline$f\left(P_{\pi}\right)$ & & 2 & & & 4 & & & & & 6 & & & & 8 \\
\hline
\end{tabular}

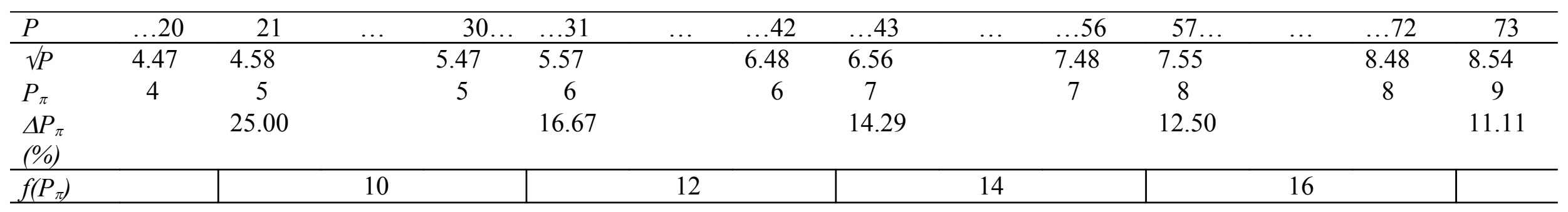

$P$ : number of publications. Number of publications in the $\pi$-core: $\mathrm{P}_{\pi}=\sqrt{\mathrm{P}}$ (rounded). $f\left(P_{\pi}\right)$ : frequency of identical $P_{\pi}$ values. $\Delta P_{\pi}(\%)$ : increase of $P_{\pi}$ in per cent. 
Table 3

Model for the increase of the $\pi$-index $(\Delta \pi \%)$ by increasing the number of papers in the $\pi$-core from $P_{\pi}=3$ to $P_{\pi m}=4$.

\begin{tabular}{llccc}
\hline Level & $\begin{array}{l}\text { Rank of } \\
\text { papers }\end{array}$ & \multicolumn{3}{c}{ Number of citations } \\
& & $\mathrm{A}$ & $\mathrm{B}$ & $\mathrm{C}$ \\
& $\mathrm{P}_{1}$ & 80 & 40 & 10 \\
& $\mathrm{P}_{2}$ & 10 & 20 & 10 \\
\hline$\pi$-level: & $\mathrm{P}_{3}$ & 10 & 10 \\
$(\pi+1)$-level & $\mathrm{P}_{4}$ & 2 & 6 & 10 \\
\hline & $\mathrm{P}_{5}$ & 6 & 10 \\
& $\mathrm{P}_{6}$ & 1 & 5 & 10 \\
& $\mathrm{P}_{7}$ & 1 & 5 & 10 \\
& $\mathrm{P}_{8}$ & 1 & 4 & 10 \\
& $\mathrm{P}_{9}$ & 1 & 3 & 10 \\
& $\mathrm{P}_{10}$ & 1 & 1 & $\mathrm{c}$ \\
\hline & & 1 & $\mathrm{~b}$ & 0 \\
& $\mathrm{P}_{11}$ & $\mathrm{a}$ & 0 & 0 \\
\hline & $\mathrm{P}_{12}$ & 0 & 0 & 0 \\
& $\mathrm{P}_{13}$ & 0 & 0 & \\
\hline
\end{tabular}

\begin{tabular}{|c|c|c|c|c|c|c|}
\hline & $\mathrm{A}$ & $\mathrm{B}$ & $\mathrm{C}$ & $\mathrm{A}+\mathrm{a}^{\prime}$ & $\mathrm{B}+\mathrm{b}^{\prime}$ & $\mathrm{C}+\mathrm{c}^{\prime}$ \\
\hline$P_{\pi}$ & 3 & 3 & 3 & & & \\
\hline$P_{\pi m}$ & & & & 4 & 4 & 4 \\
\hline$C\left(P_{\pi}\right)$ & 92 & 70 & 30 & & & \\
\hline$C\left(P_{\pi m}\right)$ & & & & 94 & 76 & 40 \\
\hline$\pi$ & 0.92 & 0.70 & 0.31 & & & \\
\hline$\pi_{m}$ & & & & 0.94 & 0.76 & 0.40 \\
\hline$\Delta \pi \%$ & & & & 2.17 & 8.57 & 29.03 \\
\hline
\end{tabular}

The number of papers in the $\pi$-core, $P_{\pi}: \mathrm{A}=\mathrm{B}=\mathrm{C}=3$ and in $P_{\pi m}: \mathrm{A}+\mathrm{a}^{\prime}=\mathrm{B}+\mathrm{b}^{\prime}=\mathrm{C}+\mathrm{c}^{\prime}=$ 4.

Total number of papers $=\mathrm{P} ; \mathrm{P}_{\pi}=\sqrt{\mathrm{P}}$

$P_{\pi}\left(\mathrm{P}_{1}-\mathrm{P}_{10}\right)=\sqrt{\mathrm{P}}=\sqrt{ } 10=3.33 \sim 3$

$P_{\pi m}\left(\mathrm{P}_{1}-\mathrm{P}_{13}\right)=\sqrt{ } \mathrm{P}=\sqrt{ } 13=3.61 \sim 4$

$\Delta \pi \%=100\left(\pi_{m}-\pi\right) / \pi$ 
Table 4

Impact of increasing the citedness of upper limit papers, C(UL) on the CDS index from $C(U L)$ to $C(U L)+1$.

\begin{tabular}{lcccccccc}
\hline \multirow{2}{*}{ Score } & \multicolumn{8}{c}{ Citedness category } \\
\cline { 2 - 9 } & 1 & 2 & 3 & 4 & 5 & $6 \ldots 13$ & \multicolumn{1}{c}{14} \\
\hline$C(L L)$ & 0 & $2^{0}+1$ & $2^{2}+1$ & $2^{3}+1$ & $2^{4}+1$ & $2^{5}+1$ & $2^{12}+1$ & $>2^{13}$ \\
$C(U L)$ & $2^{0}$ & $2^{2}$ & $2^{3}$ & $2^{4}$ & $2^{5}$ & $2^{6}$ & $2^{13}$ & \\
\hline$C(L L)$ & 0 & 2 & 5 & 9 & 17 & 33 & 4097 & $>8192$ \\
$C(U L)$ & 1 & 4 & 8 & 16 & 32 & 64 & 8192 & - \\
Score & 1 & 2 & 3 & 4 & 5 & 6 & 13 & 14 \\
$C(U L)+1$ & 2 & 5 & 9 & 17 & 33 & 65 & 8193 & - \\
Modified & 2 & 3 & 4 & 5 & 6 & 7 & 14 & 14 \\
Score & & & & & & & & \\
\hline Change \% & 100.00 & 50.00 & 33.30 & 25.00 & 20.00 & 16.67 & 0.08 & 0.00 \\
\hline
\end{tabular}

$C(L L)$ : number of citations obtained to the publication on the lower limit.

$C(U L)$ : number of citations obtained to the publication on the upper limit. 
Table 5

Model for demonstrating the change of the $h$-index by increasing the citedness of the paper ranked as $r_{(h+l)}$ in the Hirsch-core by unity.

\begin{tabular}{|c|c|c|c|c|c|}
\hline & $\begin{array}{l}\text { Rank of } \\
\text { papers } \\
(P)\end{array}$ & \multirow[t]{2}{*}{$\frac{\mathrm{a}}{\text { citations }}$} & \multirow[t]{2}{*}{$\frac{\mathrm{b}}{(\mathrm{a}+1) \text { citation }}$} & \multicolumn{2}{|c|}{$\begin{array}{l}\text { Lowest number of } \\
\text { citations in the } h \text {-core } \\
\qquad\left(\Sigma \mathrm{C}_{\text {min }}\right)\end{array}$} \\
\hline & & & & $\mathrm{a}$ & $\mathrm{b}$ \\
\hline \multirow[t]{4}{*}{$\mathrm{A}$} & $\overline{r_{h}}$ & $\overline{c_{1} \geq 1}$ & $c_{1} \geq 1$ & & \\
\hline & $r_{(h+1)} \quad 2$ & $c_{2}=0$ & $c_{2}=1$ & & \\
\hline & 3 & $c_{3}=0$ & $c_{3}=0$ & & \\
\hline & $h$-index & 1 & 1 & 1 & 1 \\
\hline \multirow[t]{4}{*}{$\bar{B}$} & $r_{h}$ & $c_{1} \geq 2$ & $c_{1} \geq 2$ & & \\
\hline & $r_{(h+1)}$ & $c_{2}=1$ & $c_{2}=2$ & & \\
\hline & 3 & $c_{3}=0$ & $c_{3}=0$ & & \\
\hline & $h$-index & 1 & 2 & 2 & 4 \\
\hline \multirow[t]{4}{*}{$\mathrm{C}$} & 1 & $c_{1} \geq 3$ & $c_{1} \geq 3$ & & \\
\hline & $r_{h}$ & $c_{2} \geq 3$ & $c_{2} \geq 3$ & & \\
\hline & $r_{(h+1)} \quad 3$ & $c_{3}=2$ & $c_{3}=3$ & & \\
\hline & $h$-index & 2 & 3 & 6 & 9 \\
\hline \multirow[t]{5}{*}{$\mathrm{D}$} & 1 & $c_{1} \geq 4$ & $c_{1} \geq 4$ & & \\
\hline & 2 & $c_{2} \geq 4$ & $c_{2} \geq 4$ & & \\
\hline & $r_{h}$ & $c_{3} \geq 4$ & $c_{3} \geq 4$ & & \\
\hline & $r_{(h+1)} \quad 4$ & $c_{4}=3$ & $c_{4}=4$ & & \\
\hline & $h$-index & 3 & 4 & 12 & 16 \\
\hline
\end{tabular}




\section{Table 6}

Simple models for demonstrating the change of the original value of the indices studied ( $\pi, C D S, h, J P C, A, R)$ by increasing the number of citations by unity $\left(\pi_{m}, C D S_{m}, h_{m}, J P C_{m}, A_{m}, R_{m}\right)$.

\begin{tabular}{|c|c|c|c|c|c|c|c|c|c|c|c|c|}
\hline \multirow[b]{2}{*}{$\begin{array}{c}\text { Original } \\
\text { value of } \\
\text { the } \\
\text { index }\end{array}$} & \multicolumn{2}{|c|}{$\pi_{m}^{a}$} & \multicolumn{2}{|c|}{$\mathrm{CDS}_{\mathrm{m}}^{\mathrm{b}}$} & \multicolumn{2}{|c|}{$h_{m}^{c}$} & \multicolumn{2}{|c|}{$\mathrm{JPC}_{\mathrm{m}}^{\mathrm{d}}$} & \multicolumn{2}{|c|}{$\mathrm{A}_{\mathrm{m}}^{\mathrm{e}}$} & \multicolumn{2}{|c|}{$\mathrm{R}_{\mathrm{m}}^{\mathrm{f}}$} \\
\hline & index & $\begin{array}{c}\text { change } \\
(\%)\end{array}$ & index & $\begin{array}{c}\text { change } \\
(\%)\end{array}$ & index & $\begin{array}{c}\text { change } \\
(\%)\end{array}$ & index & $\begin{array}{c}\text { change } \\
(\%)\end{array}$ & index & $\begin{array}{c}\text { change } \\
(\%)\end{array}$ & index & $\begin{array}{c}\text { change } \\
(\%)\end{array}$ \\
\hline 0.10 & 0.11 & 10.0 & - & - & - & - & 0.2 & 100.0 & - & - & - & - \\
\hline 0.50 & 0.51 & 2.0 & - & - & - & - & 0.6 & 20.0 & - & - & - & - \\
\hline 1.00 & 1.01 & 1.0 & 2.00 & 100.0 & 2 & 100.0 & 1.1 & 10.0 & 2.00 & 100.0 & 1.41 & 41.00 \\
\hline 2.00 & 2.01 & 0.5 & 3.00 & 50.0 & 3 & 50.0 & 2.1 & 5.0 & 2.50 & 25.0 & 2.24 & 12.00 \\
\hline 5.00 & 5.01 & 0.2 & 6.00 & 20.0 & 6 & 20.0 & 5.1 & 2.0 & 5.20 & 4.0 & 5.10 & 2.00 \\
\hline 10.00 & 10.01 & 0.1 & 11.00 & 10.0 & 11 & 10.0 & 10.1 & 1.0 & 10.10 & 1.0 & 10.05 & 0.50 \\
\hline 30.00 & 30.01 & 0.03 & 31.00 & 3.3 & 31 & 3.3 & 30.1 & 0.3 & 30.03 & 0.1 & 30.02 & 0.07 \\
\hline 50.00 & 50.01 & 0.02 & 51.00 & 2.0 & 51 & 2.0 & 50.1 & 0.2 & 50.02 & 0.04 & 50.01 & 0.02 \\
\hline 100.00 & 100.01 & 0.01 & 101.00 & 1.0 & 101 & 1.0 & 100.1 & 0.1 & 100.01 & 0.01 & 100.005 & 0.005 \\
\hline 200.00 & 200.01 & 0.005 & 201.00 & 0.5 & 201 & 0.5 & 200.1 & 0.05 & 200.005 & 0.025 & 200.003 & 0.001 \\
\hline
\end{tabular}

a: The increase of the $\pi$-index is possible only if a paper within the $\pi$-core $\left(P_{\pi}=\sqrt{ } P\right.$, where $P$ is the total number of papers, and the papers are ranked in decreasing rank number of citations) obtains at least one more citation.

${ }^{\mathrm{b}}$ : Applying the maximum value of the citedness of papers within the corresponding category (i.e.: 1, 4, 8, 16, etc., see Table 4).

c: The increase of the h-index is possible only if a paper outside the Hirsch-core will be cited (see Table 7).

d: $\mathrm{JPC}=C / P($ e.g. $1 / 10=0.1 ; 2 / 10=0.2 ; 100 / 10=10.0 ; 101 / 100=10.1)$.

e: $C\left(P_{h}\right)=h^{2} ; \mathrm{A}=C\left(P_{h}\right) / h ;\left(\right.$ e.g.: $\left.\mathrm{h}=5, \mathrm{C}\left(\mathrm{P}_{\mathrm{h}}\right)=25=\mathrm{h}^{2} ; \mathrm{A}=25 / 5=5 ; \mathrm{C}\left(\mathrm{P}_{\mathrm{h}}\right)+1=26 ; \mathrm{A}_{\mathrm{m}}=26 / 5=5.2\right)$

f: $C\left(P_{h}\right)=h^{2} ; \mathrm{R}=\sqrt{ } \mathrm{h}^{2}=\mathrm{h} ;\left(\right.$ e.g. $\left.\mathrm{h}=10, C\left(P_{h}\right)=100=\mathrm{h}^{2} ; \mathrm{R}=\sqrt{ } 100=10 ; \mathrm{C}\left(\mathrm{P}_{\mathrm{h}}\right)+1=101 ; \mathrm{R}_{\mathrm{m}}=\sqrt{ } 101=10.05\right)$ 
Table 7

Model for demonstrating the consequences of changing the citation rate of the paper ranked as $r_{h}$ and $r_{(h+1)}$ in anomalous and specific Hirsch-sets.

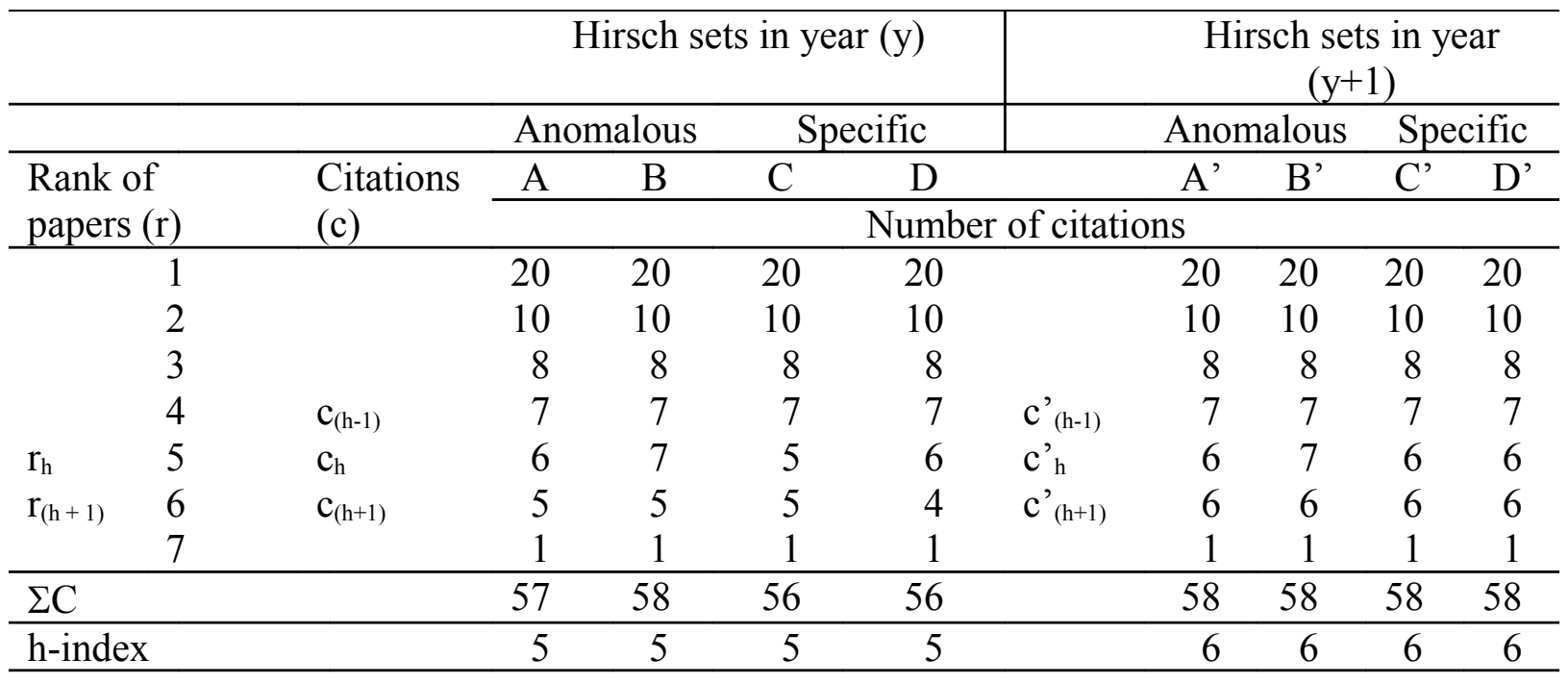

\begin{tabular}{ll}
\hline Anomalous set $(A)$ & Specific set $(\mathrm{C})$ \\
\hline $\mathrm{c}_{\mathrm{h}}=\mathrm{h}+1<\mathrm{c}_{(\mathrm{h}-1)}$ & $\mathrm{c}_{\mathrm{h}}=\mathrm{c}_{\mathrm{h}+1}=\mathrm{h}<\mathrm{c}_{(\mathrm{h}-1)}$ \\
$6=5+1<7$ & $5=5=5<7$ \\
$\mathrm{c}_{(\mathrm{h}+1)}=\mathrm{h}$ & \\
$5=5$ &
\end{tabular}

\begin{tabular}{lll}
\hline & Anomalous set $(\mathrm{B})$ & Specific set (D) \\
\hline & $\begin{array}{l}\mathrm{c}_{\mathrm{h}}=\mathrm{h}+2=\mathrm{c}_{(\mathrm{h}-1)} \\
7=5+2=7\end{array}$ & $\begin{array}{l}\mathrm{c}_{\mathrm{h}}=\mathrm{h}+1<\mathrm{c}_{(\mathrm{h}-1)} \\
6=5+1<7\end{array}$ \\
& $\begin{array}{l}\mathrm{c}_{(\mathrm{h}+1)}=\mathrm{h} \\
5=5\end{array}$ & $\begin{array}{l}\mathrm{c}_{(\mathrm{h}+1)}=\mathrm{h}-1 \\
4=5-1\end{array}$ \\
\hline $\begin{array}{l}\text { Minimum number of } \\
\text { citations to increase the } \\
\text { h-index by unity }\end{array}$ & \multicolumn{1}{c}{1} & 2 \\
\hline $\begin{array}{l}\Sigma \mathrm{C}: \text { total citations. } \\
\end{array}$ & & \\
\end{tabular}


Table 8

Model for the increase of A and R index (see Table 1) with increasing the number of citations to the $h$-core.

\begin{tabular}{|c|c|c|c|c|c|c|c|c|}
\hline \multirow{2}{*}{ Example } & \multicolumn{4}{|c|}{ Number of citations $(C)$ to the individual publications $\left(\mathrm{P}_{\mathrm{i}}\right)$} & \multirow{2}{*}{$\begin{array}{l}\text { Total number of citations } \\
\text { in the } h \text {-core } \mathrm{C}\left(\mathrm{P}_{\mathrm{h}}\right)\end{array}$} & \multirow{2}{*}{$h$} & \multirow{2}{*}{ A } & \multirow{2}{*}{$\mathrm{R}$} \\
\hline & $C\left(P_{1}\right)$ & $C\left(P_{2}\right)$ & $C\left(P_{3}\right)$ & $C\left(P_{4}\right)$ & & & & \\
\hline $\mathrm{A} / 1$ & 1 & 0 & 0 & 0 & 1 & 1 & 1.0 & 1.00 \\
\hline$A^{\prime} / 1$ & 2 & 0 & 0 & 0 & 2 & 1 & 2.0 & 1.41 \\
\hline$A^{\prime} / 2$ & 2 & 1 & 1 & 1 & 2 & 1 & 2.0 & 1.41 \\
\hline $\mathrm{A} / 3$ & 100 & 1 & 1 & 0 & 100 & 1 & 100.0 & 10.00 \\
\hline$A^{\prime} / 3$ & 101 & 1 & 1 & 0 & 101 & 1 & 101.0 & 10.05 \\
\hline $\mathrm{B} / 2$ & 3 & 3 & 2 & 1 & 6 & 2 & 3.0 & 2.45 \\
\hline$B^{\prime} / 2$ & 4 & 3 & 2 & 1 & 7 & 2 & 3.5 & 2.65 \\
\hline $\mathrm{C} / 1$ & 3 & 3 & 3 & 3 & 9 & 3 & 3.0 & 3.00 \\
\hline$C^{\prime} / 1$ & 4 & 3 & 3 & 3 & 10 & 3 & 3.3 & 3.16 \\
\hline $\mathrm{C} / 2$ & 4 & 4 & 4 & 3 & 12 & 3 & 4.0 & 3.46 \\
\hline$C^{\prime} / 2$ & 5 & 4 & 4 & 3 & 13 & 3 & 4.3 & 3.61 \\
\hline
\end{tabular}


Table 9

The Successively Built-up Indicator model (SBI-model). Possible relationships between the increase in publications and citations and the scientometric indicators (Table 1). Under $\mathrm{P}_{1}, \mathrm{P}_{2}$, etc. ' 1 ' denotes a citation to the respective publication.

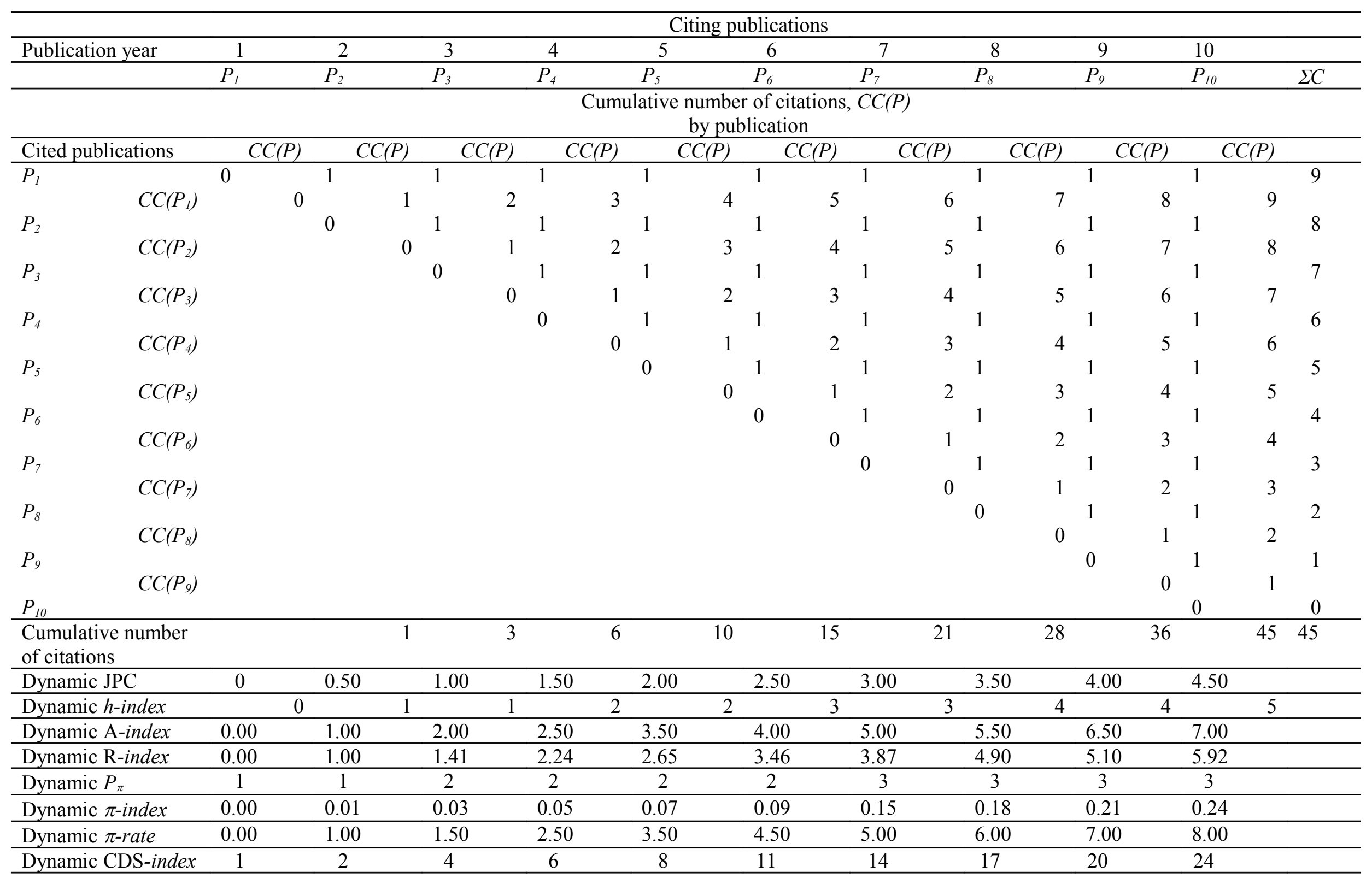


$\Sigma C$ : total number of citations obtained. 
Table 10

The scientometric impact indices calculated by the Successively Built-up Indicator (SBI) model (see Table 9) (a/: for a total of 10 papers; b/: for a total of 20 papers) as the percentage values of the mean index of junior and senior scientists. (All scientists are active in chemistry.)

\begin{tabular}{|c|c|c|c|c|c|c|c|c|c|c|c|}
\hline & & PLT & $P$ & $C$ & $C / P$ & $h$ & $\pi$ & CDS & $\pi$-rate & $\mathrm{A}$ & $\mathrm{R}$ \\
\hline $\begin{array}{l}\text { Junior } \\
\text { scientists (JS) }\end{array}$ & $\mathrm{m}$ & 8.60 & 25.30 & 297.30 & 12.66 & 8.60 & 1.90 & 54.80 & 38.20 & 24.49 & 14.95 \\
\hline $\begin{array}{l}(\mathrm{JS} / \\
(\mathrm{n}=15)\end{array}$ & SD & 2.91 & 14.31 & 209.09 & 10.15 & 2.91 & 1.29 & 31.17 & 28.46 & 9.74 & 5.31 \\
\hline $\begin{array}{l}\text { Senior } \\
\text { scientists (SS) }\end{array}$ & $\mathrm{m}$ & 32.30 & 95.60 & 1729.10 & 19.08 & 21.70 & 7.92 & 240.50 & 85.60 & 54.25 & 33.97 \\
\hline$(n=15)$ & SD & 4.27 & 31.30 & 544.77 & 6.86 & 3.62 & 2.88 & 76.30 & 38.37 & 17.19 & 7.00 \\
\hline SBI & $\mathrm{a} /$ & 10 & 10 & 45 & 4.50 & 5 & 0.24 & 24 & 8.00 & 7.00 & 5.92 \\
\hline & $\mathrm{b} /$ & 20 & 20 & 190 & 9.50 & 10 & 1.45 & 67 & 17.5 & 14.50 & 12.04 \\
\hline & \multicolumn{11}{|c|}{ Percentage ratio } \\
\hline $100(\mathrm{SBI})$ & $\mathrm{a} /$ & 116.30 & 39.53 & 15.14 & 35.54 & 58.14 & 12.63 & 43.80 & 20.94 & 28.58 & 39.60 \\
\hline JS & $\mathrm{b} /$ & 232.56 & 79.05 & 63.91 & 75.04 & 116.28 & 76.32 & 122.26 & 45.81 & 59.21 & 80.54 \\
\hline $100(\mathrm{SBI})$ & $\mathrm{a} /$ & 30.96 & 10.46 & 2.60 & 23.58 & 23.04 & 3.03 & 9.98 & 9.35 & 12.90 & 17.43 \\
\hline SS & $\mathrm{b} /$ & 61.92 & 20.92 & 10.99 & 49.79 & 46.08 & 18.31 & 27.86 & 20.44 & 26.73 & 35.44 \\
\hline
\end{tabular}

$n$ : number of scientists involved.

PLT: Publication Life Time: (2011 - the year of the first publication in WoS since 1975)

The difference between the mean of the indices calculated for junior and senior scientists are highly significant $(p<0.01)$, except for the $(C / P)$ indexes. 
Table 11

Pearson's correlation coefficients between some scientometric indices of the set consisting of 15 junior and 15 senior scientists.

\begin{tabular}{|c|c|c|c|c|c|c|c|c|c|c|}
\hline & PLT & $P$ & $C$ & $(C / P)_{t}$ & $\pi$-rate & $h$ & A & $\mathrm{R}$ & CDS & $\pi$ \\
\hline$P$ & $0.76^{*}$ & 1.00 & $0.88^{*}$ & 0.15 & 0.37 & $0.87 *$ & $0.58^{*}$ & $0.74^{*}$ & $0.99 *$ & $0.71^{*}$ \\
\hline$C$ & $0.81 *$ & $0.88 *$ & 1.00 & $0.51 *$ & $0.72 *$ & $0.96^{*}$ & $0.86^{*}$ & $0.96^{*}$ & $0.94 *$ & $0.94 *$ \\
\hline$(C / P)_{t}$ & 0.39 & 0.15 & $0.51 *$ & 1.00 & $0.89 *$ & $0.47 *$ & $0.75^{*}$ & $0.68^{*}$ & 0.25 & $0.66^{*}$ \\
\hline$h$ & $0.79 *$ & $0.87^{*}$ & $0.96^{*}$ & $0.47 *$ & $0.66^{*}$ & 1.00 & $0.81^{*}$ & $0.94 *$ & $0.92 *$ & $0.88 *$ \\
\hline PLT & 1.00 & $0.76^{*}$ & $0.81 *$ & 0.39 & $0.63 *$ & $0.79 *$ & $0.74 *$ & $0.79 *$ & $0.78^{*}$ & $0.80 *$ \\
\hline A & $0.74 *$ & $0.58 *$ & $0.86^{*}$ & $0.75^{*}$ & $0.95 *$ & $0.81 *$ & 1.00 & $0.96^{*}$ & $0.66^{*}$ & $0.98^{*}$ \\
\hline$\pi$ & $0.80 *$ & $0.71^{*}$ & $0.94 *$ & $0.66^{*}$ & $0.89 *$ & $0.88^{*}$ & $0.98^{*}$ & $0.97^{*}$ & $0.78 *$ & 1.00 \\
\hline
\end{tabular}

$P$ : Number of publications in WoS between 1975-2011 October.

$C$ : number of citations (selfcitations included) in WoS between 1975 and 2011 October.

Publication Life Time: PLT $=2011$ - (the publication year of the first paper in WoS since 1975).

For $\pi$-rate, $h, \mathrm{~A}, \mathrm{R}, \mathrm{CDS}$, and $\pi$, see Table 1 .

*: significant at $\mathrm{p}<0.01$. 